\title{
A Neuromonitoring Approach to Facial Nerve Preservation During Image-guided Robotic Cochlear Implantation
}

\author{
*Juan Ansó, †Cilgia Dür, *Kate Gavaghan, $\ddagger$ Helene Rohrbach, \\ *Nicolas Gerber, *Tom Williamson, §Enric M. Calvo, \|Thomas Wyss Balmer, \\ $\ddagger$ Christina Precht, §Damien Ferrario, \Matthias S. Dettmer, \#Kai M. Rösler, \\ $\dagger$ Marco D. Caversaccio, *Brett Bell, and *Stefan Weber
}

* ARTORG Center for Biomedical Engineering; †Department of ENT, Head and Neck Surgery, Inselspital; $\downarrow$ tVetSuisse Faculty, University of Bern, Bern; §Swiss Centre for Electronics and Microtechnology, CSEM, Neuchâtel; ||Institute for Surgical Technologies and Biomechanics, ISTB; $\Upsilon$ Institute of Pathology; and \#Department of Neurology, ENMG-Station, Inselspital, University of Bern, Bern, Switzerland

Hypothesis: A multielectrode probe in combination with an optimized stimulation protocol could provide sufficient sensitivity and specificity to act as an effective safety mechanism for preservation of the facial nerve in case of an unsafe drill distance during image-guided cochlear implantation.

Background: A minimally invasive cochlear implantation is enabled by image-guided and robotic-assisted drilling of an access tunnel to the middle ear cavity. The approach requires the drill to pass at distances below $1 \mathrm{~mm}$ from the facial nerve and thus safety mechanisms for protecting this critical structure are required. Neuromonitoring is currently used to determine facial nerve proximity in mastoidectomy but lacks sensitivity and specificity necessaries to effectively distinguish the close distance ranges experienced in the minimally invasive approach, possibly because of current shunting of uninsulated stimulating drilling tools in the drill tunnel and because of nonoptimized stimulation parameters. To this end, we propose an advanced neuromonitoring approach using varying levels of stimulation parameters together with an integrated bipolar and monopolar stimulating probe.

Materials and Methods: An in vivo study (sheep model) was conducted in which measurements at specifically planned and navigated lateral distances from the facial nerve were performed to determine if specific sets of stimulation parameters in combination with the proposed neuromonitoring system could reliably detect an imminent collision with the facial nerve. For the accurate positioning of the neuromonitoring probe, a dedicated robotic system for image-guided cochlear implantation was used and drilling accuracy was corrected on postoperative microcomputed tomographic images.

Results: From 29 trajectories analyzed in five different subjects, a correlation between stimulus threshold and drillto-facial nerve distance was found in trajectories colliding with the facial nerve (distance $<0.1 \mathrm{~mm}$ ). The shortest pulse duration that provided the highest linear correlation between stimulation intensity and drill-to-facial nerve distance was $250 \mu$ s. Only at low stimulus intensity values $(\leq 0.3 \mathrm{~mA})$ and with the bipolar configurations of the probe did the neuromonitoring system enable sufficient lateral specificity $(>95 \%)$ at distances to the facial nerve below $0.5 \mathrm{~mm}$. However, reduction in stimulus threshold to $0.3 \mathrm{~mA}$ or lower resulted in a decrease of facial nerve distance detection range below $0.1 \mathrm{~mm}$ ( $>95 \%$ sensitivity). Subsequent histopathology follow-up of three representative cases where the neuromonitoring system could reliably detect a collision with the facial nerve (distance $<0.1 \mathrm{~mm}$ ) revealed either mild or inexistent damage to the nerve fascicles.

Conclusion: Our findings suggest that although no general correlation between facial nerve distance and stimulation threshold existed, possibly because of variances in patient-specific anatomy, correlations at very close distances to the facial nerve and high levels of specificity would enable a binary response warning system to be developed using the proposed probe at low stimulation currents. Key Words: Bipolar-Cochlear implant-Facial nerve-Image-guided-MastoidectomyMonopolar-Neuromonitoring-Robotic.

Otol Neurotol 37:89-98, 2016.
Address correspondence and reprint requests to Kate Gavaghan, Ph.D., ARTORG Center for Biomedical Research, University of Bern, Murtenstrasse 50, 3008, Bern, Switzerland; E-mail: kate.gavaghan@ artorg.unibe.ch

The authors received funding from the Swiss National Science Foundation (Nano-Tera initiative, HearRestore project), the European Commission (under the Hear-EU project, grant number: 304857), and Med-EL GmbH (Innsbruck, Austria) for work related to this study.

The authors disclose no conflicts of interest.

Supplemental digital content is available in the text.
In the last decade, image-guided approaches using freehand navigation (1-3), template techniques (4-6), and robotic assistance $(7-10)$ have been advocated to reduce the invasiveness associated with mastoidectomy during conventional cochlear implantation (CI). Recently, two studies $(11,12)$ have demonstrated sufficient end-to-end image-guided drilling accuracies of $0.31 \pm 0.1$ and $0.15 \pm 0.08 \mathrm{~mm}$ using templates and a robotic guidance 
respectively, suggesting their potential clinical implementation. The minimally invasive approach is based on the creation of a small diameter tunnel $(1.5-2 \mathrm{~mm})$ from the surface of the patient's mastoid to the cochlea following a preoperatively planned path through the facial recess. The approach results in the drilling tool passing within close proximity $(0.1-1.2 \mathrm{~mm}[6,12])$ of the facial nerve (FN), placing this vital structure at risk from either excessive drilling temperature or mechanical destruction by collision with the drill bit. Although electromyogram (EMG)-based neuromonitoring has become a standard safety mechanism for identification and preservation of the FN in middle ear surgeries, such as revision cases and cochlear implantations (13-21), it has yet to achieve the sensitivity and specificity required to become a reliable safety mechanism when operating in close vicinity of the FN $(<0.3 \mathrm{~mm})$, without line of sight to the surrounding anatomy.

In an initial investigation toward the integration of a continuous monopolar neuromonitoring drill with a surgical robot system (22), a lack of sensitivity and specificity to detect if the FN lies within an unsafe vicinity of the drill was determined, possibly because of current shunting of the uninsulated drill shaft (cathode electrode) with the walls of the direct cochlear access (DCA) tunnel. Several studies have analyzed the effects of uninsulated versus insulated electrodes as well as the effects of monopolar versus bipolar stimulation. Kartush and Prass $(23,24)$ suggest use of insulated stimulation probes (monopolar or bipolar) to avoid current shunting in the presence of conducting fluids.

The use of a monopolar insulated stimulation tool is predicted to provide high detection sensitivity, but may be associated with a reduced lateral specificity. Conversely, bipolar stimulation that provides a more focused stimulation pattern may provide greater spatial selectivity at close ranges to the FN, but possibly insensitivity at larger distances. To overcome this dilemma, a combined monopolar and bipolar stimulation approach enabling a tradeoff between sensitivity and specificity is proposed. We hypothesize that a multielectrode insulated stimulation tool in combination with an optimized stimulation protocol could provide sufficient sensitivity and specificity to act as an effective safety mechanism for preservation of the $\mathrm{FN}$ in case of an unsafe distance of the drill (e.g., $<0.3 \mathrm{~mm}$ ).

Herein, we present a multipolar parameterized stimulation approach on the basis of a custom-made probe that can be used to avoid an impending collision between the drill and the FN during minimally invasive imageguided CI. To validate that spatial selectivity (sensitivity and specificity) of FN detection can be increased using the focused conduction geometry at the tool tip combined with varying levels of current intensity and stimulus duration, an in vivo animal study was conducted. Results of the study, which involved the drilling of tunnels at various controlled distances to the FN, stimulation and subsequent recording of muscular response, correlated with microcomputed tomographic $(\mu \mathrm{CT})$ images and histopathological follow-up, are presented. On the basis of the determined EMG to FN distance relationship, a safety mechanism stimulation protocol for use in minimally invasive cochlear implantation is proposed.

\section{MATERIALS AND METHODS}

\section{Neuromonitoring System}

A multielectrode stimulation probe for monopolar and bipolar FN monitoring was developed. It consists of a cone-like cathode ( $h=0.3 \mathrm{~mm}, r=0.3 \mathrm{~mm})$ and three stainless-steel ringlike anodes (1.8-mm diameter, 1-mm axial cross section) distributed distally along the shaft at distances $d=2,4$, and $7 \mathrm{~mm}$ (Fig. 1). The probe provides three bipolar configurations for focused stimulation in front of the tool tip, and a monopolar configuration offering higher penetration depth. To maximize electrode-tissue contact inside the direct cochlear access tunnel, the probe was designed to replicate the geometry of the drill bit used in the robotic approach.

A stimulator (STMISOLA, Biopac Systems, Inc., Goleta, CA, U.S.A.) was used to provide a predefined current waveform between the cathode and anode electrodes. To allow the current source to be switched among the four cathodeanode configurations, a relay was implemented. Stimulus response was recorded using a configurable amplification
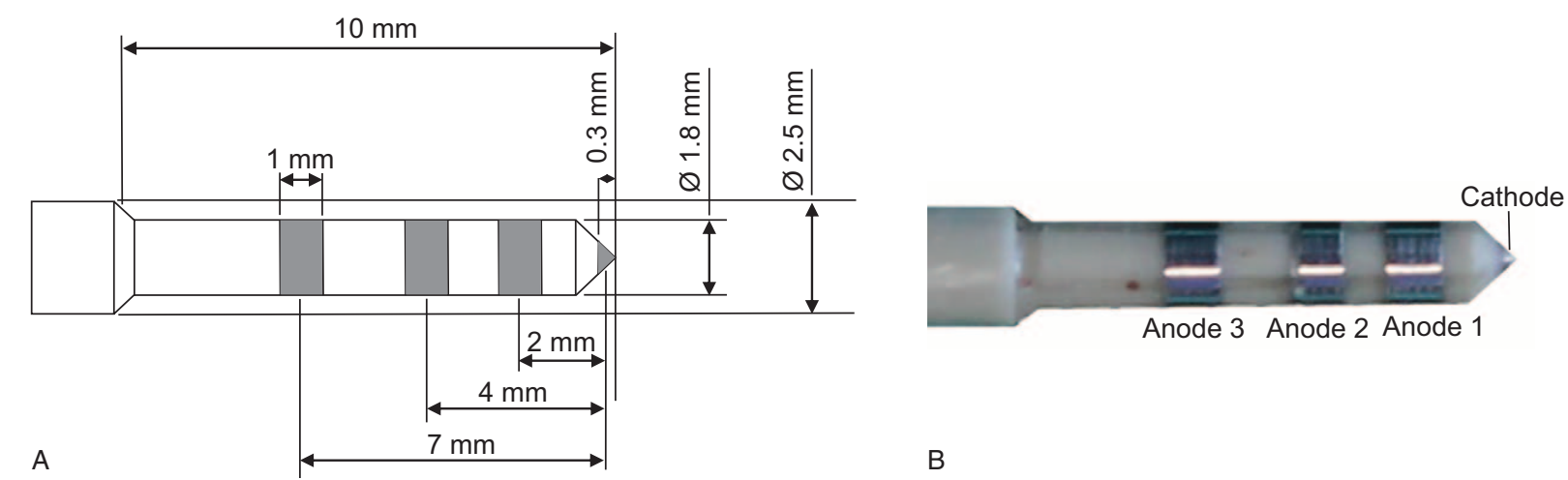

FIG. 1. Schematic $(A)$ and implemented multielectrode probe $(B)$ with the cathode electrode at the tip and the three ring-like anodes along the shaft at distances $d=2,4$, and $7 \mathrm{~mm}$. 
system (MP150, EMG100C, Biopac Systems) at $20 \mathrm{kHz}$ with 16-bit resolution. For verification of electrode impedance values, a general-purpose amplifier (DA100C, Biopac, U.S.A.) was used.

\section{In Vivo Experiment}

With approval of the local institutional review board (Bernese cantonal animal commission, approval number 57/12) the developed probe and stimulation protocol were tested in five animals. A sheep model was chosen, because, despite the existence of air-filled tympanic bulla and the nonexistence of air cells (25), it provides similar mastoid and FN anatomy to human (26).

\section{Anesthesia Protocol}

Animals were desensitized with $0.1 \mathrm{mg} / \mathrm{kg}$ diazepam and $0.1 \mathrm{mg} / \mathrm{kg}$ butorphanol administered intravenously. General anesthesia was induced with an intravenous injection of thiopental $2.5 \%$. Endotracheal intubation was performed, whereby the anesthetic state was maintained by isoflurane in $100 \%$ oxygen. Ringers lactate solution was administered at a rate of $10 \mathrm{ml} / \mathrm{kg} / \mathrm{h}$. To ensure normal FN activity, no neuromuscular blockade was used.

\section{Imaging, Access, and Measurement Planning}

The temporalis muscle was excised and four titanium reference screws ( $2.2 \mathrm{~mm}$ diameter $\times 5 \mathrm{~mm}$ length, REF M-5243.05, Medartis, Basel, Switzerland) were implanted near the external auditory canal for later registration of the physical and image spaces (as described by Gerber et al. (27)). A CT image $\left(0.2 \times 0.2 \times 0.4 \mathrm{~mm}^{3}\right)$ of the animal's head was acquired (Brilliance, Philips AG, Amsterdam, The Netherlands) and drilling trajectories $(1.8 \mathrm{~mm} \varnothing)$ were planned relative to the segmented facial nerve using a modified version of the otological surgical planning software described by Gerber et al. (28). Three groups of three parallel trajectories were defined with lateral distances (LD) of $0.5,0.25$, and $0.0 \mathrm{~mm}$ from the $\mathrm{FN}$, for each animal. Four measurement points representing decreasing closest Euclidian distances (CD) $(0.25-\mathrm{mm}$ increments) from the drill bit to the FN were defined on the axis of each trajectory (Fig. 2). An additional fifth measurement point was defined $0.5 \mathrm{~mm}$ axially from measurement point 4 in noncolliding trajectories, and $0.25 \mathrm{~mm}$ in colliding trajectories. Although in an image-guided robotic cochlear implantation a lateral distance to the facial nerve of $0.0 \mathrm{~mm}$ would neither be planned nor expected, the experimental nature of this study enables differences in correlation of facial nerve distance to stimulus threshold intensity for a frontal and a lateral collision to be investigated.

\section{Drilling and Facial Nerve Stimulation Measurements}

Two subdermal EMG electrodes were inserted into the Orbicularis Oris and Oculi muscles and a ground electrode was placed central to the nose bridge. A stimulation-return electrode (monopolar configuration) was positioned in the mastoid region contralateral to the surgical site. The EMG
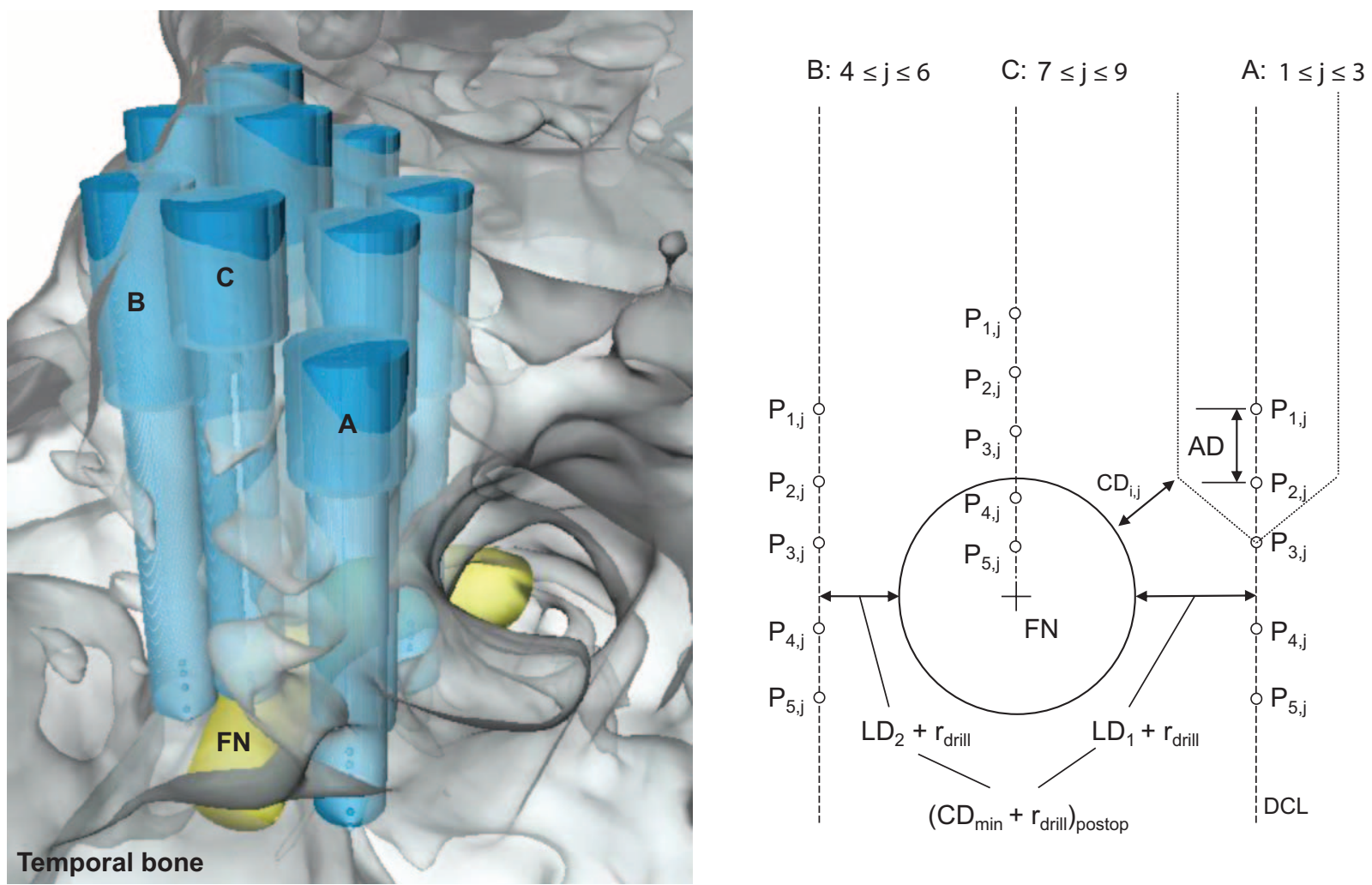

FIG. 2. The three groups of planned trajectories with target positions defined relative to the FN. $(A)$ The first group of trajectories with a lateral distance $\mathrm{LD}_{1}=0.5 \mathrm{~mm}$. $(B)$ The second group with a lateral distance $\mathrm{LD}_{2}=0.25 \mathrm{~mm}$. $(C)$ The third group approaching the FN frontally $(\mathrm{LD}=0 \mathrm{~mm})$. The trajectory number is denoted by $j$ The probe tip represented at one measurement point $\left(\mathrm{P}_{3, j}\right)$ through the drilled center line (DCL), with the closest distance to the $\mathrm{FN}$ denoted as $C \mathrm{CD}_{\mathrm{i}, \mathrm{j}}$. The minimum postoperative measured distance from the tunnel to the $\mathrm{FN}$ along each trajectory is denoted $C D_{\min }$. 
and impedance amplifiers were set to a gain of 500 and 1 respectively, and a frequency bandwidth of 0.01 to $5 \mathrm{kHz}$. An active optical reference marker was attached to the probe to allow its pose to be tracked inside the DCA tunnel by the robotic system's optical tracking camera.

After immobilization of the animal's head using a pin type head-clamp, a high-accuracy image-guided surgical robot system $(12,22,29,30)$ was used to drill to each of the predefined measurement points in the planned trajectories (see Video, Supplemental Digital Content 1, http://links.lww.com/MAO/ A345, which shows how the image-guided robotic system drills to the next measurement point). Upon reaching a given measurement position, the drill was removed, and to ensure good electrical contact between electrodes and bony tissue, irrigation $(\mathrm{NaCl}, 0.9 \%)$ was applied. At each measurement position, a stimulation sequence of 40 monophasic pulses at $4 \mathrm{~Hz}(\geq 5 \mathrm{~mA}$, duration $=250 \mu \mathrm{s})$ was applied between two surface electrodes on the superficial branch of the FN trunk, serving as a positive control for FN sensitivity. Electrode-tissue impedance was measured $(1 \mathrm{kHz})$ between each electrode pair. Then, monophasic pulses $(4 \mathrm{~Hz})$ with varying durations $(50$, $100,150,250$, and $500 \mu \mathrm{s})$ and amplitudes (10 logarithmically spaced values from 0.1 to $1 \mathrm{~mA}$ for the monopolar and $d=7$ configurations, and between 0.3 and $3 \mathrm{~mA}$ for the $d=2$ and $d=4$ probe configurations) were applied and the two EMG channels recorded for each pulse duration and electrode pair. Upon completion of all measurements, subjects were euthanized and the mastoid removed and preserved in formalin for postoperative analysis.

\section{DATA ANALYSIS}

\section{Microcomputed Tomographic Facial Nerve Distance Calculation}

Mastoids were imaged using micro-CT with an isometric resolution of $18 \mu \mathrm{m}$ (Scanco $\mu \mathrm{CT} 40$, Scanco Medical, Briittisellen, Switzerland) and tunnels and the FN were manually segmented (Amira, FEI, Hillsboro, OR, U.S.A.). Spatial positions of the measurement points $\left(P_{i}\right)$ were determined along the segmented tunnel axes (DCL) and the distances between the tunnel and the FN were measured. The smallest distance measured between each drilled tunnel and the FN was defined as the minimum closest distance $\mathrm{CD}_{\min }$ (Fig. 2).

\section{Distance to EMG Stimulus Threshold Analysis}

EMG responses were examined in a 25-ms search window after a 2.1-ms rejection period after the stimulation pulse. The presence of artifacts (e.g., isotonic discharges) at the level of the electrically induced electromyogram signals led to exclusion of the contaminated channel from further analysis. The minimum current intensity that produced an EMG response was defined as the stimulus threshold (ST). Linear regression of recorded stimulus thresholds was used to determine the existence and strength of a correlation for each of the employed pulse durations and electrode configurations. To analyze the effect of lateral distance on the stimulus-distance relationship, only the ST values of measurement points approaching the $\mathrm{FN}\left(P_{1}-P_{4}\right)$ were considered and trajectories were grouped into four categories: safe lateral (LD >0.5 mm), high risk lateral $(0.1<\mathrm{LD}<0.5 \mathrm{~mm})$, lateral collision $(\mathrm{LD}<0.1 \mathrm{~mm})$, and frontal collision (LD $<0 \mathrm{~mm}$ and DCL intersecting the $\mathrm{FN}$ ).

Sensitivity and Specificity of Facial Nerve Detection

Using the minimal pulse duration providing a linear correlation (Pearson's correlation coefficient $r \geq 0.8$ ) between ST and FN distance, a sensitivity and specificity analysis of FN safety detection for seven stimulus intensity thresholds $\left(I_{i}=0.1,0.2,0.3,0.4,0.5,1.0,1.5 \mathrm{~mA}\right)$ was carried out. The minimum distance from the drilled path to the $\mathrm{FN}\left(\mathrm{CD}_{\min }\right)$ of each trajectory was compared with a safety margin distance denoted as FN distance threshold ( $D_{i}=0.1$ to $1 \mathrm{~mm}, 0.1-\mathrm{mm}$ steps $)$. Positive and negative FN detection rates were identified for each of the 10 distance thresholds (Di), depending on existence or lack of an EMG response (see Table, Supplemental Digital Content 2, http://links.lww.com/MAO/A346, which illustrates the classification criteria), and rates for true positive (sensitivity) and true negative (specificity) were computed.

\section{Safe Facial Nerve Distance Detection}

Using the sensitivity and specificity curves calculated as a function of the $\mathrm{FN}$ distance margin $\left(D_{i}\right)$, reliable versus unreliable neuromonitoring intervals were defined as: 1) safe zone, where the lack of an EMG response with a specificity $>95 \%$ indicates a sufficient distance margin between the drilled tunnel and the FN; 2) unsafe zone, where a positive stimulus response indicates an unsafe distance of the drilled tunnel and the FN with a sensitivity $>95 \%$; 3) uncertainty zone, where sensitivity $<95 \%$ and specificity $<95 \%$ prevent a reliable detection of FN proximity.

\section{Assessment of Facial Nerve Damage}

To assess the level of FN damage in patients where the facial canal was possibly penetrated (FN distance $<0.1 \mathrm{~mm}$ ), histopathology of the extracted temporal bones of subjects 2 and 4 was performed. After a conventional histopathological work-up at the center of each tunnel, the level of injury was assessed. A slice containing a completely destroyed FN plane was prepared as a control sample of total FN impairment.

\section{RESULTS}

A total of 42 trajectories were drilled in five subjects, each tunnel consisting of five measurement positions at varying depths. Difficulties to determine the location of the FN in the postoperative imagery lead to the exclusion of 12 of the initial 42 tunnels. From the electrode-tissue impedance measures realized before each neuromonitoring probe stimulation, only one trajectory of the remaining 30 presented an unexpectedly high value $(>100 \mathrm{k} \Omega$ ). The neuromonitoring measurements of that trajectory were determined to be unreliable, and thus excluded from further analysis (total exclusion of 13/42 tunnels). 
The mean electrical impedance of the remaining cases $(\mathrm{n}=29$ trajectories $\times 4$ electrode pairs $\times 5$ measurement points) was $5.7 \pm 2.2 \mathrm{k} \Omega$. These values quantify electrode-tissue contact quality once the probe is inside the tunnel, but do not directly express any measure of remaining electrode-tissue impedance from the tip of the probe to the facial nerve.

Of the 29 trajectories included in the final analysis, 15 tunnels did not collide with the FN, eight with a lateral distance above $0.5 \mathrm{~mm}$, and seven passing between 0.1 and $0.5 \mathrm{~mm}$. Finally, a total of 14 tunnels had a distance to the FN below $0.1 \mathrm{~mm}$, with 4 of them classified as lateral collisions and 10 as frontal collisions.

\section{Correlation of Stimulus Threshold to Facial Nerve Distance}

Correlations between stimulus threshold (ST) values and the closest tunnel-to-FN distance were only found in trajectories that approached the $\mathrm{FN}$ with a lateral distance of less than $0.1 \mathrm{~mm}$. As depicted in Figure 3, the change in required stimulus intensity related to $\mathrm{FN}$ distance (steepness of the fitting curves) was greater for frontal than for lateral collision cases. For the rest of the analysis the $250-\mu$ s duration is used as it was found to be the shortest pulse providing the highest linear correlation $(r=0.8, p<0.001)$ among most electrode configurations. From the fitting linear model, ST values at the FN boundary representing the minimal required current for FN stimulation were found to be between 0.2 and $0.3 \mathrm{~mA}$.

The stimulus threshold curves along the drill axis and relative to the FN (in the $\mu \mathrm{CT}$ images) of nine representative trajectories are illustrated in Figure 4. Interestingly, the frontal $(\mathrm{A}-\mathrm{C})$ and lateral collision $(\mathrm{D}-\mathrm{F})$ examples show stimulus threshold values of 0.1 to $0.3 \mathrm{~mA}$ at the fallopian canal for the monopolar and the bipolar electrode pairs. In Figure 4H and I, two examples of trajectories with lateral distances above $0.3 \mathrm{~mm}$ expressing dehiscence (a connecting channel) are shown. The acute drop of current at the point at which the tip of the probe contacted the channel suggests that changes in bone density relative to the position of the stimulating electrode will influence the level of intensity required to elicit an EMG response. Such channels remain undetectable in imaging at resolution levels applicable to clinical routine

\section{Sensitivity and Specificity of Facial Nerve Detection}

FN detection with $100 \%$ sensitivity was achieved for an unsafe distance to the $\mathrm{FN}$ defined at $0.3 \mathrm{~mm}$ at stimulus intensities above $1 \mathrm{~mA}$ (see Figure a, Supplemental Digital Content 3, http://links.lww.com/MAO/ A347, which illustrates the sensitivity curves of FN detection). However, the specificity of FN detection at $1 \mathrm{~mA}$ was very low $(<15 \%)$, indicating a large amount of false-positive EMG responses (see Figure b, Supplemental Digital Content 3, http://links.lww.com/MAO/ A347, which illustrates the specificity curves of FN detection). The probe configuration $d=2$ provided $100 \%$ specificity at $0.3 \mathrm{~mA}$, whereas the monopolar was most specific at $0.1 \mathrm{~mA}$. For small ST values $(0.3$ and $0.2 \mathrm{~mA}$ ) the sensitivity increased with decreasing distance to the FN (see Figure c, d, Supplemental Digital Content 3, http://links.lww.com/MAO/A347, with the sensitivity and specificity curves as a function of $\mathrm{FN}$ distance), reaching a $100 \%$ true positive rate below $0.1 \mathrm{~mm}$ to the FN. Specificity remained $100 \%$ at any FN distance for the bipolar electrode configuration $(d=2)$ at $0.3 \mathrm{~mA}$, and for the $d=7$ configuration at $0.2 \mathrm{~mA}$. The monopolar configuration at $0.2 \mathrm{~mA}$ only produced $100 \%$ specificity at FN distances above $0.5 \mathrm{~mm}$.
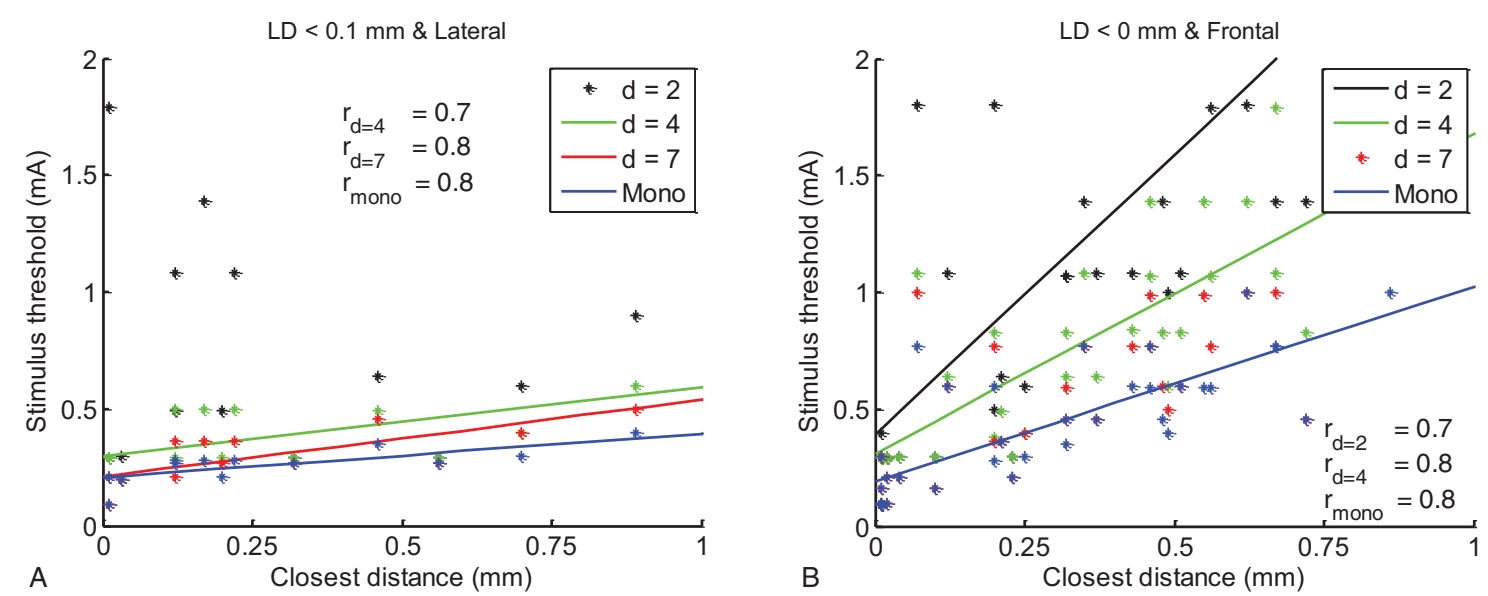

FIG. 3. Scatter plot representing examples of stimulus threshold values relative to FN distance for the duration $=250 \mu \mathrm{S}$ and trajectories with a lateral distance to the FN below $0.1 \mathrm{~mm}$. The fitting lines represent the electrode pairs which produced a positive correlation with $r \geq$ $0.7(p<0.05)$. ( $A)$ In the lateral collision group, the monopolar (blue line) and $d=7$ (red line) configurations produced the highest correlation values $(r=0.8, p<0.001)$. (B) In the frontal collision trajectories, the monopolar and the $d=4$ electrode pairs had the highest linear correlations $(r=0.8)$. From the linear fitting models, it was found that a frontal trajectory required approximately 2.5 times higher intensities as compared with lateral collisions (e.g., $1 \mathrm{~mA}$ vs $0.4 \mathrm{~mA}$ at $1 \mathrm{~mm}$ distance to the $\mathrm{FN}$ for the monopolar configuration). 
Frontal collision examples

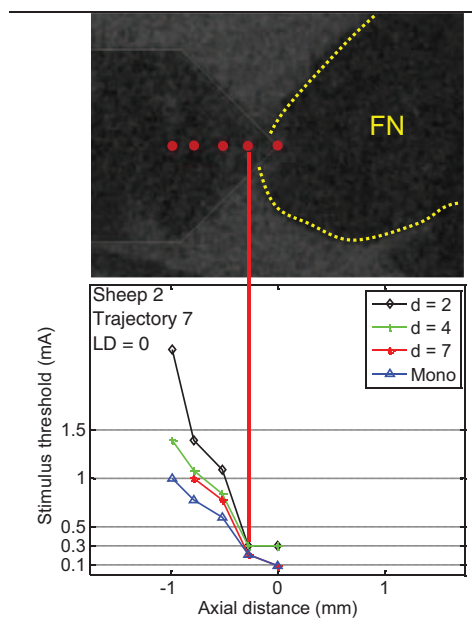

A

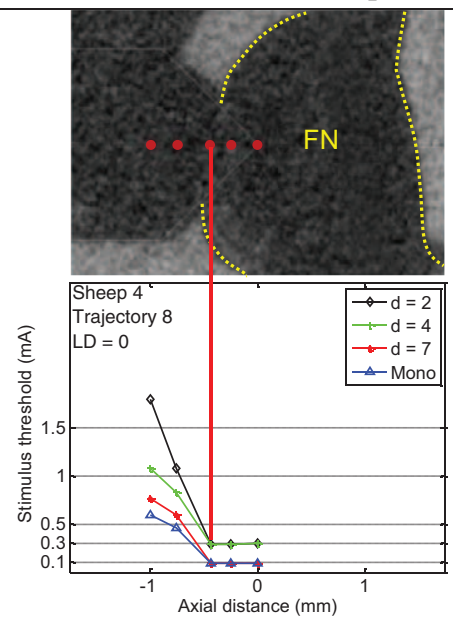

B

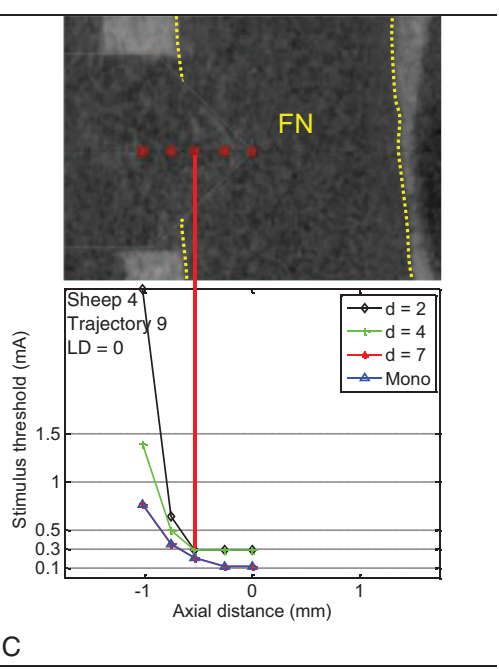

Lateral collision examples

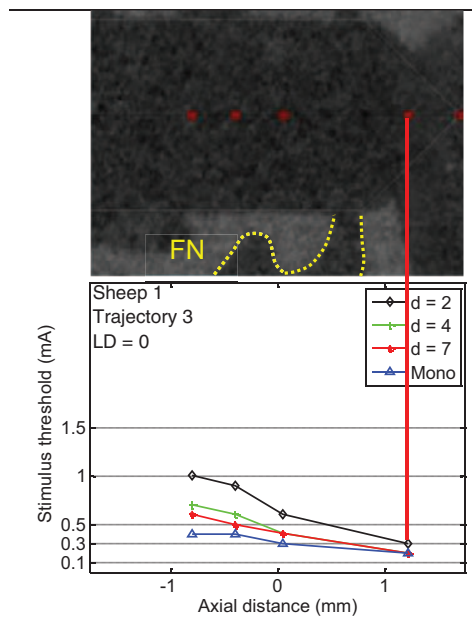

D

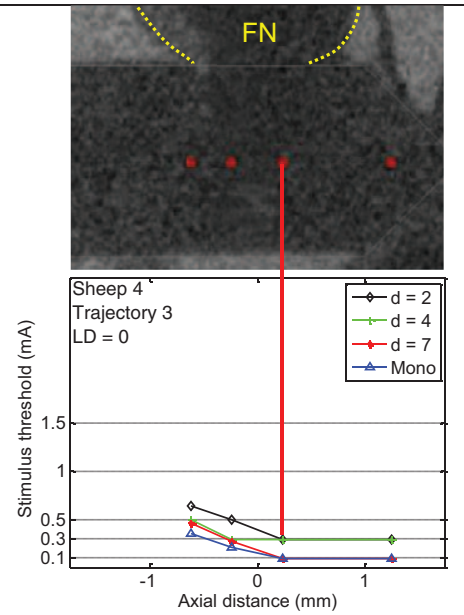

E
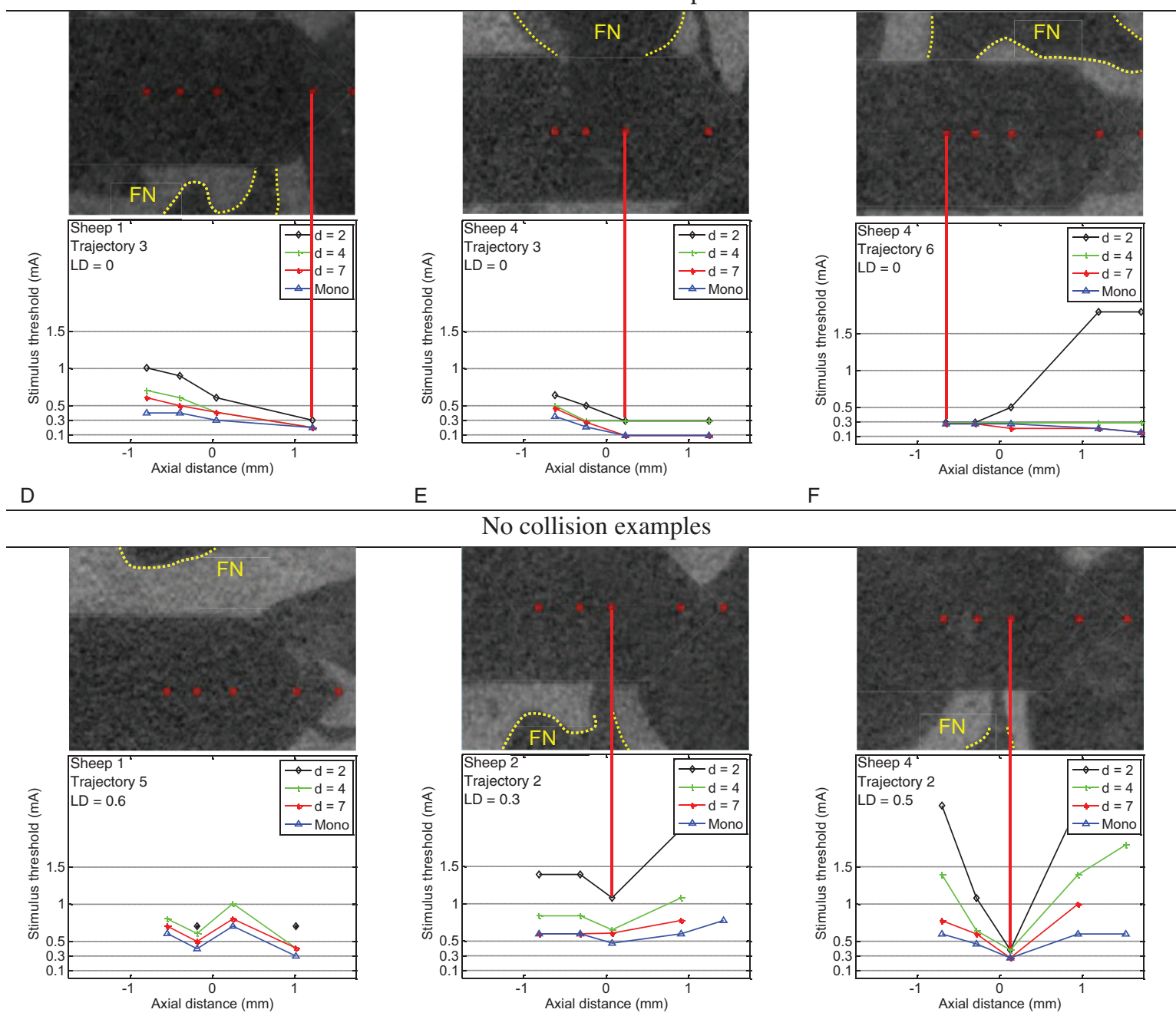

G

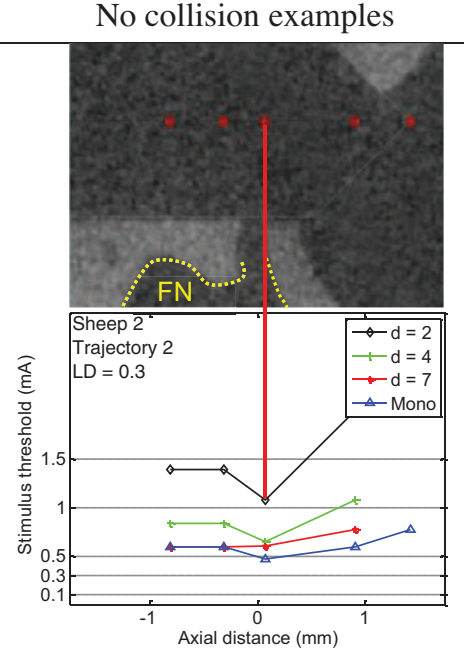

H

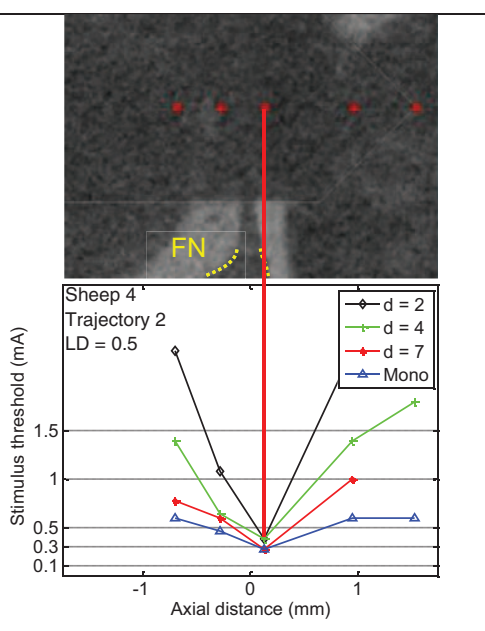

FIG. 4. Representative examples of stimulus threshold responses classified according to different lateral distance groups: $(A-C)$ Examples of frontal collisions showing larger drops of stimulus threshold values relative to FN distance; $(D-F)$ Lateral collisions cases with stimulus thresholds below $0.3 \mathrm{~mA}$ warning of an imminent collision with the canal; $(G-l)$ Examples of no collision trajectories with lateral distances to the $\mathrm{FN}$ above $0.3 \mathrm{~mm}$. 
Definition of Safe and Unsafe Drilling Zones

In Figure 5, the determined safe and unsafe zones for varying stimulation intensities and electrode configurations are depicted in green and red, whereas the white regions represent uncertainty zones. Only at stimulus intensities below $0.4 \mathrm{~mA}$ did the neuromonitoring system enable sufficient specificity and sensitivity at distances between 0.1 and $0.4 \mathrm{~mm}$ to the facial nerve. At $0.3 \mathrm{~mA}$, the monopolar electrode increased the sensitive range of FN detection from 0.1 to $0.3 \mathrm{~mm}$, which shifted the lateral specificity range away from the FN $(>0.7 \mathrm{~mm})$. Further increase in stimulus intensity above $0.4 \mathrm{~mA}$ expanded the sensitivity range, whilst diminishing specificity. At $1 \mathrm{~mA}$, the probe was always able to stimulate the FN; thus it was unable to identify FN distance.

\section{Histopathology Inspection of Facial Nerve Damage}

Representative trajectories that collided frontally or laterally $(<0.1 \mathrm{~mm})$ with the $\mathrm{FN}$ are represented in Figure 6. In the top panels (A and D), a smaller magnification allows the drilled tunnels relative to the $\mathrm{FN}$ in the mastoid to be observed. On the left column $(\mathrm{A}-\mathrm{C})$, the middle trajectory (subject2-trajectory7) penetrated the fallopian canal and provoked a localized hemorrhage with fibrin exudation (orange arrow). The FN is locally damaged (hypereosinophilic structures) next to the region with hemorrhage. A damaged fascicle (black arrow) represents the major component of injury, as compared with the untouched healthy FN regions (green arrow). Extrapolating the position of the tip of the drill bit relative to the FN, the damaged fascicles were likely not to be because of direct contact of the cutting tool, but because of pressure caused by the bleeding or drilling forces. Avoiding the last drilling step into the fallopian canal (Fig. 4A) could have minimized the pressure to those nerve fascicles. Two collision cases (trajectories 9 and 6) of subject 4 are represented in panels D-F. In the frontal collision (S4-T9), the histopathology slice depicts no FN damage (green arrows). The trajectory passing next to the FN (S4-T6) leaved only a thin (approximately $20 \mu \mathrm{m}$ ) layer of vital bone with respect to the FN (blue arrow), which remained untouched (green arrow).

\section{DISCUSSION}

Within this work, a potentially clinical applicable and effective implementation of a neuromonitoring-based FN protective system for a minimally invasive approach to the cochlea has been presented. Experimental results demonstrated that although unable to provide a sufficiently accurate continuous estimation of drill to FN distance, possibly because of the effects of patientspecific anatomical variance in the mastoid region surrounding the $\mathrm{FN}$, the presented system could still reliably provide a binary response indication of nerve safety.

Unlike other previously described system (22), use of the proposed neuromonitoring probe and protocol with low levels of stimulation intensity $(0.2-0.3 \mathrm{~mA})$ reliably detected collisions of the drill bit with the FN within distances from the fallopian canal below $0.1 \mathrm{~mm}$. This retrospective discretization of the available drill to FN distance into safe and non-safe zones with sensitivity and specificity values above $95 \%$ could allow hazardous collisions of the drill bit with the FN to be avoided in future studies. Although the narrow stopping distance range of $\mathrm{FN}$ detection $(<0.1 \mathrm{~mm})$ cannot ensure the fallopian canal remains untouched, histopathology examination indicated that even with slight penetration of the fallopian canal, an immediate stop of robotic operation may prevent axonal injury of the facial nerve.
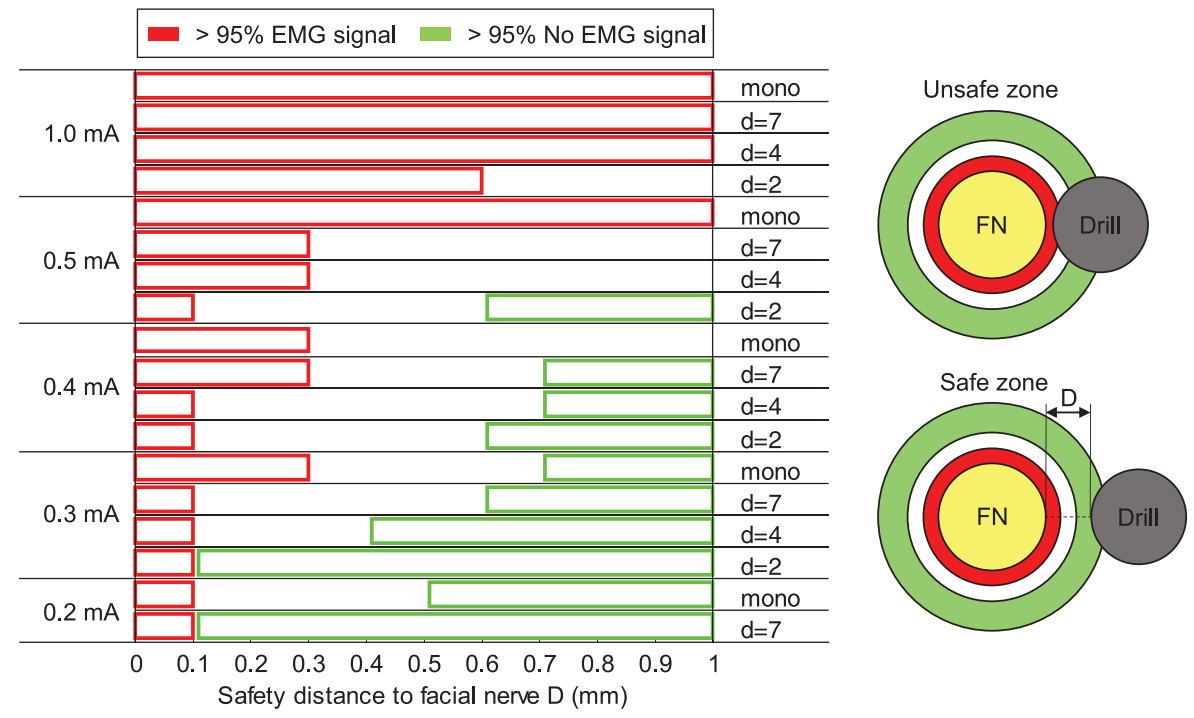

FIG. 5. Per electrode configuration and for different stimulus intensity values, the resulting FN distance detection intervals with sensitivity and specificity above $95 \%$ are represented. The FN distance regions where sensitivity and specificity were below $95 \%$ are left in white. High lateral specificity and sensitivity at distances to the FN below $0.3 \mathrm{~mm}$ were only possible with the electrode pairs $d=2$ and $d=7$ at the stimulus intensities of 0.3 and $0.2 \mathrm{~mA}$, respectively. 


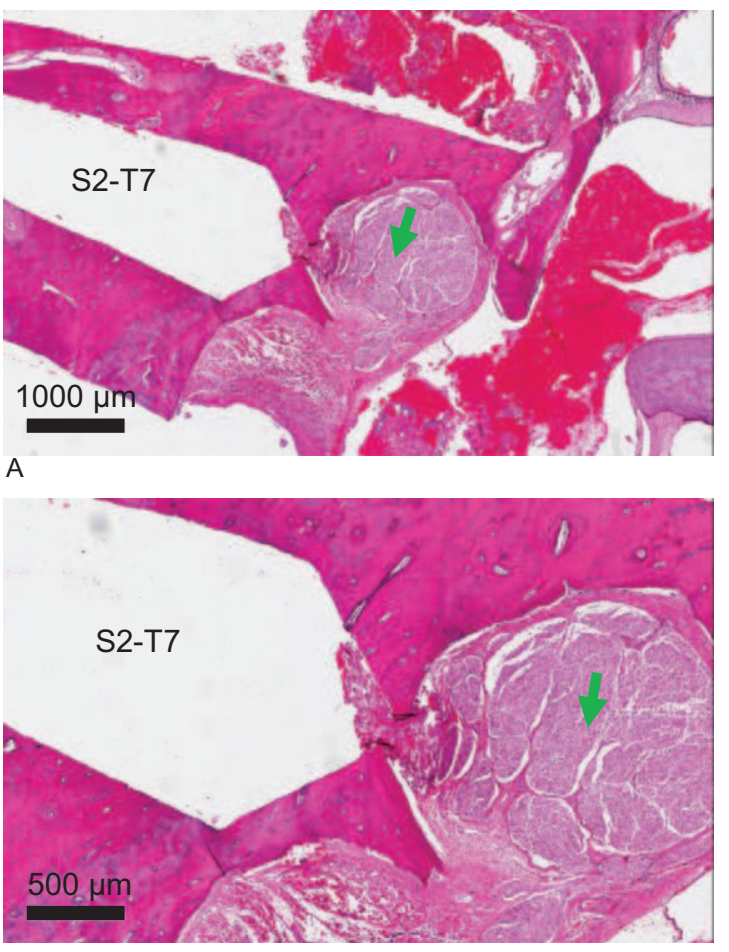

B

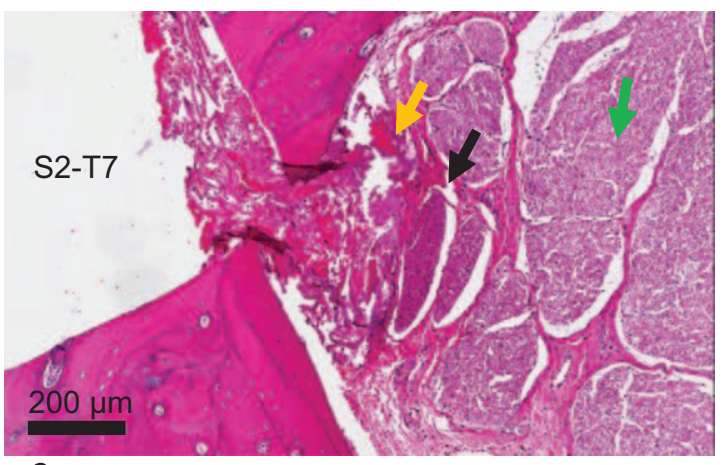

C

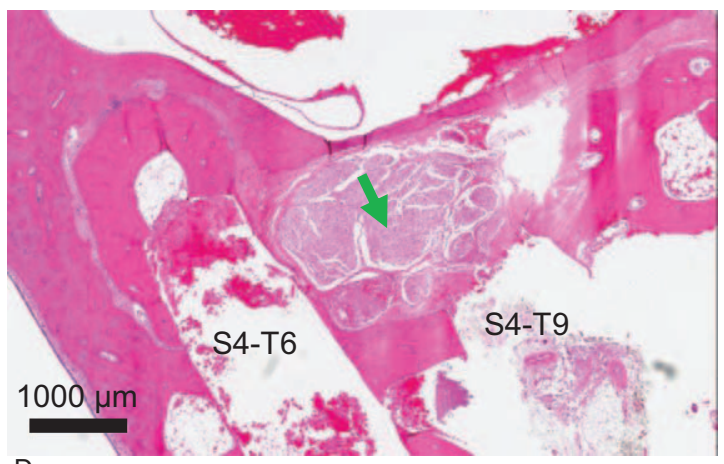

D

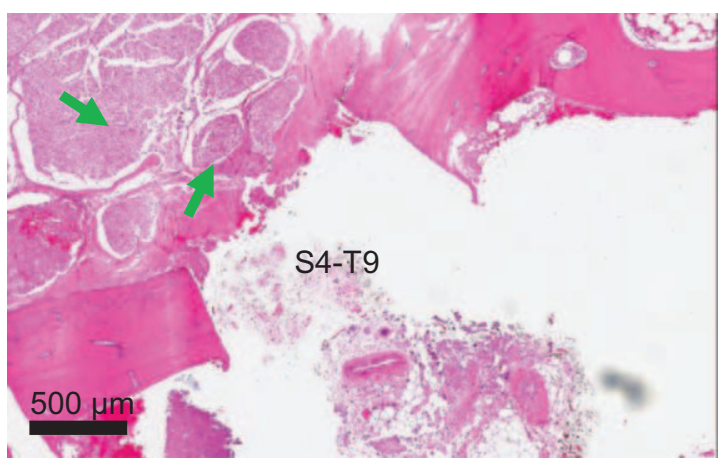

E

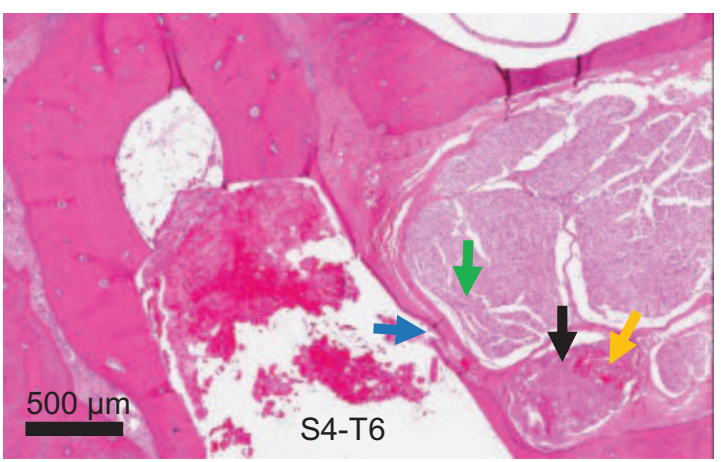

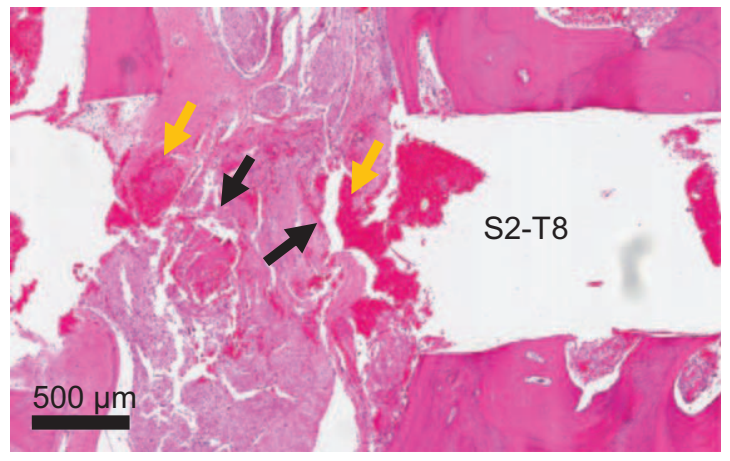

G

FIG. 6. Histopathological examples representative of collision cases between the drilling tool and the FN. Two columns are presented with different magnifications of the trajectories of interest. In the bottom panel $(G)$, an example showing the degree of FN damage produced in case of a fatal drilling through the $\mathrm{FN}$ (intentionally done once in every subject at the end of the neuromonitoring protocol). ( $A-C$ ) The middle trajectory produced local FN damage, likely because of a hemorrhage into a nerve fascicle and consecutive increased pressure (yellow and black arrows). (D-E) A frontal collision example (S4-T9) with no apparent injury of any fascicles in the region of the drilled tunnel (green arrow). $(F)$ A lateral tunnel (S4-T6) with an unsafe FN distance $(<0.1 \mathrm{~mm})$, which did not produce any trauma to the FN fascicles $(\mathrm{green}$ arrow). The black and orange arrows next to S4-T6 (in F) indicate a damaged FN fascicle. 
Because of the high-specificity requirements of minimally invasive cochlear implantation, intensities of $0.3 \mathrm{~mA}$ (or lower) combined with the proposed neuromonitoring system are recommended to identify a collision with the FN. The monopolar electrode configuration used with intensities from 0.5 to $1 \mathrm{~mA}$ (as in conventional mastoidectomy) produced an unacceptable false positive rate that could result in a $100 \%$ rejection rate of imageguided CI surgeries. However, this high sensitivity of FN detection could be used as an initial course identification of FN proximity, before initialization of a more specific detection protocol involving lower levels of stimulation current (e.g., $\leq 0.3 \mathrm{~mA}$ ) in combination with the bipolar configurations of the probe.

In agreement with results presented by Silverstein et al., this study demonstrated that a 1-mA monopolar stimulation could detect the FN at approximately $1-\mathrm{mm}$ distance (see the linear fitting model in Fig. 3). However, at closer distances, the lack of a precise stimulus intensity to FN distance relationship agreed with a comparable study from Follett and Mann (31). This could possibly be because of the large influence of patientspecific anatomy on stimulus threshold values, as it was revealed in the acute drops of stimulus intensities observed when the probe contacted liquid filled channels connecting with the fallopian canal (Fig. 4, H and I). This effect on stimulus intensity variance suggests that neuromonitoring calibration algorithms based on patientspecific bone density information could be investigated in the future to further improve the accuracy of FN detection.

Within the presented study, the use of a stimulating probe instead of drill-integrated monitoring resulted in a limited number of measurement points (5) per drilled trajectory and thus limitations in the spatial resolution of the drill-to-FN distance mapping. For future investigations, we advocate that direct stimulation while drilling could enable similar levels of lateral specificity while maintaining or improving the sensitivity of unsafe FN distance detection. However, the introduction of a rotatory tool may decrease the stability of the stimulus-evoked EMG data and thus, intermittent monitoring of the electrical impedance (electrode-tissue contact) during the drilling process is recommended.

In conclusion, the results of this in vivo study advocate that the newly developed probe and protocol could be used in combination with strategic step drilling in the neighborhood of the $\mathrm{FN}$ in minimally invasive cochlear implantation to prevent damage to the FN. However, although the very narrow FN distance detection range of $0.1 \mathrm{~mm}$ provided by the neuromonitoring probe may prevent mechanical damage, it does not ensure the FN remains preserved in terms of possible high temperatures during drilling, as described in (11). Therefore, it is proposed that the described system be used in conjunction with other reported methods of drilling error detection such as the use of intraoperative imaging or redundant instrument tracking algorithms (29). Finally, although stimulation intensity values found in the sheep model proved similar to those obtained on patients (21), translation of the proposed system and protocols to human subjects requires further verification.

Acknowledgments: The authors acknowledge Prof. Thomas Nevian for guidance with electrophysiology aspects related to the neuromonitoring protocol. The authors also thank Mark Siegrist for realization of micro-CT scans of the temporal bone samples collected during the in vivo study. The authors thank Prof. Martin Frenz for scientific contributions relating to the published work.

\section{REFERENCES}

1. Schipper J, Klenzner T, Aschendorff A, et al. [Navigation-controlled cochleostomy. Is an improvement in the quality of results for cochlear implant surgery possible?]. HNO 2004;52:329-35.

2. Labadie RF, Chodhury P, Cetinkaya E, et al. Minimally invasive, image-guided, facial-recess approach to the middle ear: Demonstration of the concept of percutaneous cochlear access in vitro. Otol Neurotol 2005;26:557-62.

3. Warren FM, Balachandran R, Fitzpatrick JM, et al. Percutaneous cochlear access using bone-mounted, customized drill guides demonstration of concept in vitro. Otol Neurotol 2007;28:325-9.

4. Labadie RF, Noble JH, Dawant BM, et al. Clinical validation of percutaneous cochlear implant surgery: Initial report. Laryngoscope 2008;118:1031-9.

5. Labadie RF, Mitchell J, Balachandran R, et al. Customized, rapidproduction microstereotactic table for surgical targeting: Description of concept and in vitro validation. Int J Comput Assist Radiol Surg 2009;4:273-80.

6. Balachandran R, Mitchell JE, Blachon G, et al. Percutaneous cochlear implant drilling via customized frames: An in vitro study. Otolaryngol Head Neck Surg 2010;142:421-6.

7. Majdani O, Rau TS, Baron S, et al. A robot-guided minimally invasive approach for cochlear implant surgery: Preliminary results of a temporal bone study. Int J Comput Assist Radiol Surg 2009;4: $475-86$.

8. Baron S, Eilers H, Munske B, et al. Percutaneous inner-ear access via an image-guided industrial robot system. Proc Inst Mech Eng Part H J Eng Med 2010;224:633-49.

9. Klenzner T, Ngan CC, Knapp FB, et al. New strategies for high precision surgery of the temporal bone using a robotic approach for cochlear implantation. Eur Arch Otorhinolaryngol 2009;266: 955-60.

10. Bell B, Stieger C, Gerber N, et al. A self-developed and constructed robot for minimally invasive cochlear implantation. Acta Otolaryngol 2012;132:355-60.

11. Labadie RF, Balachandran R, Noble JH, et al. Minimally invasive image-guided cochlear implantation surgery: First report of clinical implementation. Laryngoscope 2014;124:1915-22.

12. Bell B, Gerber N, Williamson T, et al. In vitro accuracy evaluation of image-guided robot system for direct cochlear access. Otol Neurotol 2013;34:1284-90.

13. Silverstein H, Smouha E, Jones R. Routine identification of the facial nerve using electrical stimulation during otological and neurotological surgery. Laryngoscope 1988;98:726-30.

14. Leonetti JP, Matz GJ, Smith PG, Beck DL. Facial nerve monitoring in otologic surgery: Clinical indications and intraoperative technique. Ann Otol Rhinol Laryngol. 1990;99(11):911-8. Accessed May 7, 2013.

15. Jackler RK, Selesnick SH. Indications for cranial nerve monitoring during otologic and neurotologic surgery. Am J Otol. 1994; 15(5):611-3. Accessed May 7, 2013.

16. Pensak ML, Willging JP, Keith RW. Intraoperative facial nerve monitoring in chronic ear surgery: A resident training experience. Am J Otol 1994;15:108-10. 
17. Noss RS, Lalwani a K, Yingling CD. Facial nerve monitoring in middle ear and mastoid surgery. Laryngoscope 2001;111: 831-6.

18. Wilson L, Lin E, Lalwani A. Cost-effectiveness of intraoperative facial nerve monitoring in middle ear or mastoid surgery. Laryngoscope. 2003;113(10): 1736-45.

19. Choung YH, Park K, Cho MJ, et al. Systematic facial nerve monitoring in middle ear and mastoid surgeries: "Surgical dehiscence" and "electrical dehiscence." Otolaryngol Head Neck Surg 2006;135:872-6.

20. Bernardeschi D, Meskine N, Otaibi N Al, et al. Continuous facial nerve stimulating burr for otologic surgeries. Otol Neurotol 2011;32:1347-51.

21. Heman-Ackah SE, Gupta S, Lalwani AK. Is facial nerve integrity monitoring of value in chronic ear surgery? Laryngoscope 2013;123:2-3.

22. Ansó J, Stahl C, Gerber N, et al. Feasibility of using EMG for early detection of the facial nerve during robotic direct cochlear access. Otol Neurotol 2014;35:545-54.

23. Kartush JM, Niparko JK, Bledsoe SC, et al. Intraoperative facial nerve monitoring: A comparison of stimulating electrodes. Laryngoscope 1985;95:1536-40.
24. Prass R, Lüders H. Constant-current versus constant-voltage stimulation. Neurosurgery 1985;62:622-3.

25. Seibel VAA, Lavinsky L, De Oliveira JAP. Morphometric study of the external and middle ear anatomy in sheep: A possible model for ear experiments. Clin Anat 2006;19:503-9.

26. Seibel VA, Lavinsky L, Irion K. CT-scan sheep and human inner ear morphometric comparison. Braz J Otorhinolaryngol. 2006;72(3): $370-6$.

27. Gerber N, Gavaghan K, Bell B, et al. High accuracy patient-to-image registration for the facilitation of image guided robotic microsurgery on the head. IEEE Trans Biomed Eng 2013;60:960-8.

28. Gerber N, Bell B, Gavaghan K, et al. Surgical planning tool for robotically assisted hearing aid implantation. Int J Comput Assist Radiol Surg 2014;9:11-20.

29. Williamson TM, Bell BJ, Gerber N, et al. Estimation of tool pose based on force-density correlation during robotic drilling. IEEE Trans Biomed Eng 2013;60:969-76.

30. Wimmer W, Venail F, Williamson T, et al. Semiautomatic cochleostomy target and insertion trajectory planning for minimally invasive cochlear implantation. Biomed Res Int 2014;2014:596498.

31. Follett KA, Mann MD. Effective stimulation distance for current from macroelectrodes. Exp Neurol 1986;92:75-91. 